Check for updates

Cite this: J. Mater. Chem. A, 2021, 9 , 22418

Received 24th May 2021

Accepted 18th September 2021

DOI: $10.1039 / \mathrm{d} 1 \mathrm{ta} 04367 f$

rsc.li/materials-a

\section{Enhanced ionic transport in ferroelectric polymer fiber mats $\dagger$}

\author{
Ayesha Sultana, (ID) ab Md. Mehebub Alam, (D) a Simone Fabiano, (D) a Xavier Crispin (iD *ab \\ and Dan Zhao (D)*a
}

The limited ionic conductivity is the main issue for the application of solid-state ionic conductors. In this work, we have shown that increasing the ferroelectric phase content in a polymer matrix could enhance the molar ionic conductivity of the incorporated ionic liquid by two orders of magnitude compared to the original films with the same composition. The ferroelectric polymer fiber mats were prepared through electrospinning to induce the ferroelectric phase that ensure the polarization of the dipoles. After analyzing the ferroelectric phase content and polarization of the fiber mats and films containing different ion concentration with FTIR spectroscopy and piezoelectric characterization, a detailed mechanism explaining the improved conductivity in the ferroelectric fiber mats was proposed. Benefiting from the good flexibility, improved ionic conductivity and high temperature coefficient of the fiber mats, we fabricated an organic ionic thermistor. The temperature tracking and mapping function of the ionic thermistor was demonstrated by using two devices with 4 and 16 pixels.

\section{Introduction}

Ionic conductors that transport ions have been under limelight and intensively studied in recent years because of their important function in diverse modern techniques. The unique characters of ionic conductors have enabled various novel applications such as electrolyte gated transistors ${ }^{1,2}$ ionic thermoelectric devices, ${ }^{3-5}$ and flexible electroluminescent devices. ${ }^{6,7}$ Considering the importance of the coupling between ions and electrons in intimate biological communication systems, ${ }^{8}$ nowadays, there is a trend of developing ionic modulated biologically active chemicals delivery devices ${ }^{9}$ and multifunctional wearable sensors. ${ }^{10-12}$ To improve the reliability issue and processibility of the ionic related devices, developing solid-state electrolytes with good ionic conductivity is of great importance.

Various families of polymer electrolytes have drawn attention because they are flexible, lightweight, physically stable as membranes or gels, and easy to prepare, which make them suitable for large-scale applications. ${ }^{13}$ Water-based polymer electrolytes such as hydrogels, polyelectrolytes and ionomers possess high ionic conductivity typically between 1-100 $\mathrm{mS} \mathrm{cm}{ }^{-1}$, because water promotes ionic mobility. However, the low electrochemical stability window (ESW) $(\sim 1.2 \mathrm{~V})$ due to water electrolysis is a major drawback for the stability of the

${ }^{a}$ Laboratory of Organic Electronics, Department of Science and Technology, Linköping University, SE-60174 Norrköping, Sweden. E-mail: xavier.crispin@liu.se;dan.zhao@ liu.se

${ }^{b}$ Wallenberg Wood Science Center, Linköping University, SE-601 74 Norrköping, Sweden

$\dagger$ Electronic supplementary information (ESI) available. See DOI: $10.1039 / \mathrm{d} 1 \mathrm{ta} 04367 \mathrm{f}$ electrochemical devices. ${ }^{14,15}$ In the later years, the scientific community has focused on the design of new water-free polymer electrolytes of high ESW with the challenge to obtain as high ionic conductivity as possible. Indeed, the ionic conductivity is a key parameter that affects the performance of many energy harvesting and storage devices such as fuel cells, ${ }^{16}$ solar cells, ${ }^{17}$ batteries ${ }^{18}$ and supercapacitors. ${ }^{19}$ On top of that, another key property is the flammability of the electrolyte. For safety reason, the elimination of organic solvents becomes necessary when proposing new families of polymer electrolytes. Direct incorporation of salts into polymer matrix could meet the requirements of high ESW and low flammability, however, usually lead to low conductivity (0.001-0.1 mS cm ${ }^{-1}$ at RT) ${ }^{20}$ Higher ionic conductivity $\left(\sigma=0.1-10 \mathrm{mS} \mathrm{cm}^{-1}\right.$ at RT) can be obtained yet with another family of polymer electrolytes based on ionic liquid polymer gels, ${ }^{21}$ because the introduction of ionic liquid could effectively reduce the crystallization and improve the segmental motion of the polymer. ${ }^{22}$ The latter also has a large ESW (3-4.8 V) ${ }^{23}$ and displays low flammability. ${ }^{24}$

Variety of polymers such as, hydrocarbon polymers (PEO, PVA), aromatic polymers (SPEEK, PEEK), fluorinated polymers (PVDF and its copolymers) have been demonstrated as the matrix for ionic liquid to form gels with conductivity values in the range of $\sim 10^{-6}$ to $10^{-3} \mathrm{~S} \mathrm{~cm}^{-1} .^{25}$ Several strategies have been proposed to maximize the motion of polymer chains and facilitate the ion transport, such as including plasticizer, ${ }^{26}$ increasing the free volume,${ }^{27}$ but it is partially detrimental to the mechanical property. Copolymers could solve this issue by only dissolving ionic liquid in one phase and using the other phase to maintain a property of mechanical strength. As one ideal candidate, $\mathrm{P}(\mathrm{VDF}-\mathrm{HFP})$ composed of semicrystalline PVDF 
phase that are not soluble in IL, and amorphous HFP part with large absorption ability for various ionic liquids. ${ }^{28}$ Moreover, PVDF based polymer electrolytes also have good electrochemical stability and high charge/discharge efficiency. ${ }^{29,30}$ However, despite the successful utilization of P(VDF-HFP) ionic liquid polymer electrolytes in different applications, ${ }^{3,31-33}$ the transport mechanism in such ionic conductors, especially the effect of ferroelectric nature of the PVDF has not been completely understood.

In this work, we explored the ionic transport in a series of PVDF based polymer electrolytes containing gradually increasing amount of ionic liquid when the PVDF phase has different percentage of ferroelectric phase content. Through the process of electrical poling, the ferroelectric phase content can be increased and usually leads to enhanced polarization in ferroelectric materials. ${ }^{34}$ In a blend of a ferroelectric materials with an electrolyte, the ions screen the external electric field and prevent an efficient poling of the ferroelectric domains. The proposed solution is to use electrospinning method to induce in situ electrical poling and uniaxial stretching of the fiber under the high applied electric field to align the dipoles spontaneously in PVDF based ionic liquid polymer gels (IL-PGs). ${ }^{35}$ The molar ionic conductivity of the polarized electrospun IL-PG was observed to be two orders of magnitude higher than that of the solution casted films with the same composition. By discussing the conductivity and activation energy of IL-PGs of ionic liquid content in both electrospun fiber mats and drop-casted films, we provided important information on the ionic transport mechanism and insights for the design of polymer electrolytes. Finally, taking advantage of the good ionic conductivity and mechanical strength, we demonstrated the first thin film organic ionic thermistor with high temperature coefficient of resistance (TCR). Through simple and low-cost cutting and lamination, the ionic thermistor can synchronously map the temperature distribution of a surface with multiple addressable pixels.

\section{Results and discussion}

\subsection{The preparation of the ferroelectric IL-PG fiber mats}

Electrospinning is a widely used technique to prepare different types of polyelectrolyte membranes. ${ }^{33,36-38}$ Aside from producing uniform porous structures composed of nanofibers, the strong electric field applied to the material during the fiber formation directly modulate the distribution of the ionic domains in the fibers. ${ }^{38}$ Previous studies have shown that electrospinning is an effective one step technique to polarize PVDF and copolymers, and the electrospun fibers are directly employed for piezoelectric devices. ${ }^{35,39,40}$ We used this technique to prepare ferroelectric IL-PG fiber mats composed of P(VDF-HFP) and different amount of ionic liquid. As illustrated in Fig. 1a, a high voltage is applied between the needle and the collector during electrospinning. The droplet at the tip of the needle is charged and ejects a jet when the repulsive force within the charge droplet overcomes the surface tension of the solution. The jet starts to travel in a straight-line but bend due to the instabilities. ${ }^{41}$ At this step, the stretching of fiber along electric field promotes the formation of polar $\beta$-phase in PVDF fibers by orienting the $-\mathrm{CH}_{2}-\mathrm{CF}_{2}$ dipoles in the molecular chains. ${ }^{35}$ The jet then approaches to the grounded collector along the electric field and forms a fiber mat with relatively aligned dipoles, as illustrated by the arrows in Fig. 1a. Although the fibers are randomly oriented, but dipoles are still oriented in a particular direction (along the thickness of the fiber mat) as the dipoles follow the applied high voltage during the formation of the fiber mat. ${ }^{35}$

In order to load the fiber mats with uniformly distributed ionic liquid, different amount of ionic liquid (IL) was blended with the polymer solution before electrospinning. 1-Ethyl-3methylimidazolium bis(trifluoromethylsulfonyl)imide (EMIM TFSI) was chosen because it has selective solubility in the HFP phase while not in PVDF phase. ${ }^{4}$ The $\mathrm{P}(\mathrm{VDF}-\mathrm{HFP})$ fiber mats containing $7.7 \%$ to $30 \%(\mathrm{w} / \mathrm{w})$ of EMIM TFSI were prepared through electrospinning. It is difficult to form stable jet in the electrospinning process for higher IL content due to the high ionic conductivity. ${ }^{33}$

The morphology of the fiber mats containing different amount of IL are investigated with SEM, as shown in the inset of in Fig. $1 \mathrm{~b}$ and S1. $\uparrow$ Compared to the pure P(VDF-HFP) where beaded fiber can be observed, with the addition of IL no more beads were formed. We also observed that the amount of IL affects the diameter of the fibers. As shown in Fig. 1b, the obtained fibers become thinner with small amount of IL ( $0 \%$ to $14 \%)$. This is because the increasing conductivity of the solution decreases the length of the jet, which experience more
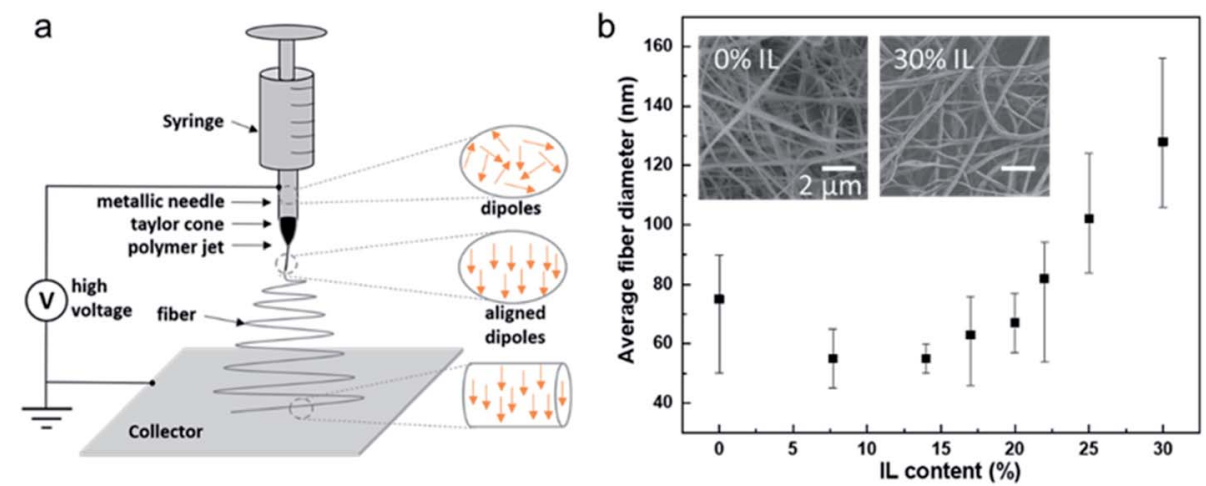

Fig. 1 Preparation of the ferroelectric fiber mats. (a) Illustration of the electrospinning process. (b) The average diameter and distribution range of the electrospun fibers with different IL content. Inset figures are the SEM images of the electrospun fiber mats containing $0 \%$ and $30 \%$ IL. 
stretching and form thin fibers due to the earlier bending instability. ${ }^{41}$ The diameter of the fibers becomes larger with further increment of IL amount (17\% to $30 \%)$ due to the "backbuilding" of the fibers ${ }^{42}$ where the fibers aggregation and fusion can be observed. The degree of fiber fusion and aggregation increases with the IL content and thus the average fiber diameter increases as shown in Fig. $1 \mathrm{~b}$.

\subsection{The characterization of the ferroelectric fiber mats}

PVDF is a semicrystalline polymer having different crystalline phases $\left(\alpha-, \beta-, \gamma^{-}\right.$, and $\delta$-phase) in which polar $\beta$-phase is the most desired phase as it exhibits maximum polarization and thus superior piezo, pyro and ferroelectric properties. ${ }^{43}$ However, non-polar $\alpha$-phase is the most stable phase in PVDF prepared through conventional procedure of melt cooling or solution casting. Thus, different post treatments such as stretching, annealing and poling are executed to transform $\alpha$ phase to $\beta$-phase. ${ }^{\mathbf{4 3 - 4 5}}$ The phase composition of the electrospun fiber mats containing different amount of IL were analyzed by FT-IR spectroscopy and compared with its solution casted films counterparts. As shown in Fig. 2a, for pure P(VDF-HFP), the peak of non-polar $\alpha$-phase at $764 \mathrm{~cm}^{-1}$ is more pronounced in the film than in the fiber mat, ${ }^{\mathbf{4 6}}$ and the peak of the electroactive phase at $841 \mathrm{~cm}^{-1}$ has the opposite trend. ${ }^{46}$ The semi-polar $\gamma$ peak at $1234 \mathrm{~cm}^{-1}$ is not present in the fiber but there is a little hump in the film,${ }^{46}$ which indicates that for the fiber, polar $\beta$ phase $\left(1275 \mathrm{~cm}^{-1}\right)$ is dominating; while for the film, ${ }^{46}$ both $\beta$ and $\gamma$-are present. The polar $\beta$-phase content of the fiber and the film is calculated to be, respectively $75 \%$ and $32 \%$ from the FTIR spectra (shown in ESI, $\uparrow$ Note S1). This is in agreement with the discussion from previous reports. ${ }^{47,48}$

The spectra of film and fiber mat after doping with IL is shown in Fig. 2b. Here, 25\% IL is used as an example, spectra for all the fiber and film composites are also given in Fig. S2. $\dagger$ For both the IL doped P(VDF-HFP) fiber mats and films, the intensity of vibrational bands corresponding to the IL (Fig. S3 $\dagger$ ) increases with increasing IL contents in the original solutions, which proves the incorporation of the IL in P(VDF-HFP) matrix. Compared to pure P(VDF-HFP) (Fig. 2a), the incorporation of the IL leads to an intensity decrease of the peak related to the non-polar $\alpha$-phase $\left(764 \mathrm{~cm}^{-1}\right)$ and an intensity increase of the peak associated to the electroactive phase $\left(841 \mathrm{~cm}^{-1}\right)$ in both $\mathrm{IL}$ doped films and fiber mats. The resulted increasing of electroactive phases is a typical effect of adding IL to P(VDF-HFP) due to the electrostatic interaction between the ions and dipoles. ${ }^{49}$ Although the total electroactive phase ( $\beta$ - plus $\gamma$-phase) in composite films are similar to the fiber mats (75 $\pm 5 \%$ ), the polar $\beta$-phase in the films $(30 \pm 4 \%)$ is much lower than that in fiber mats $(88 \pm 3 \%$ ) (Fig. 2c). The FTIR characterization of films and fiber mats follow the description in previous report and show similar results that the ferroelectric $\beta$-phase content in electrospun fiber is higher than that in the film. ${ }^{39}$ It is well accepted that higher content of $\beta$-phase indicates higher dipole orientation, which also results in improvement of piezoelectric property of the fiber. ${ }^{50}$ The increase in polar $\beta$-phase in the electrospun fiber mats after adding IL is due to the increased conductivity in the solution that leads to higher stretching of the fibers, which agrees with the morphology and diameter changes observed in SEM images.

\subsection{The ionic transport in ferroelectric fiber mats}

The ionic transport in the IL-PG fiber mats and films were investigated for various IL content. The ionic conductivity of polymer electrolytes depends on the concentration of mobile ions and their mobility along with polymer segmental mobility or polymer chain mobility. ${ }^{\mathbf{5 1}}$ The conductivity of all the films and fiber mats are shown in Fig. S4. $\dagger$ Interestingly, although the porosity of the fiber mats is around $77 \% \pm 5$ (measurement and calculation details given in ESI, Note S2 $\dagger$ ), the conductivity of the ferroelectric mats is higher than the films for the same IL content. In order to remove the concentration effect, the molar conductivity at room temperature $\left(22^{\circ} \mathrm{C}\right)$ of the films and fiber mats are calculated and compared in Fig. 3 a (calculation details in ESI, Note $\mathrm{S} 3 \dagger$ ). For the films (yellow dots), the gradually increasing molar conductivity with IL content indicates an improvement in the mobility of the ions. This is due to the increasing amount of IL plasticizes the polymer and forms better conductive percolation pathways for ionic transport in the polymer matrix. ${ }^{52}$ The molar conductivity of the fiber mats (blue squares in Fig. 3a) increases first with IL contents, and decreases after reaching the maximum value at $25 \%$ of IL content. Importantly, the molar conductivity of the ferroelectric
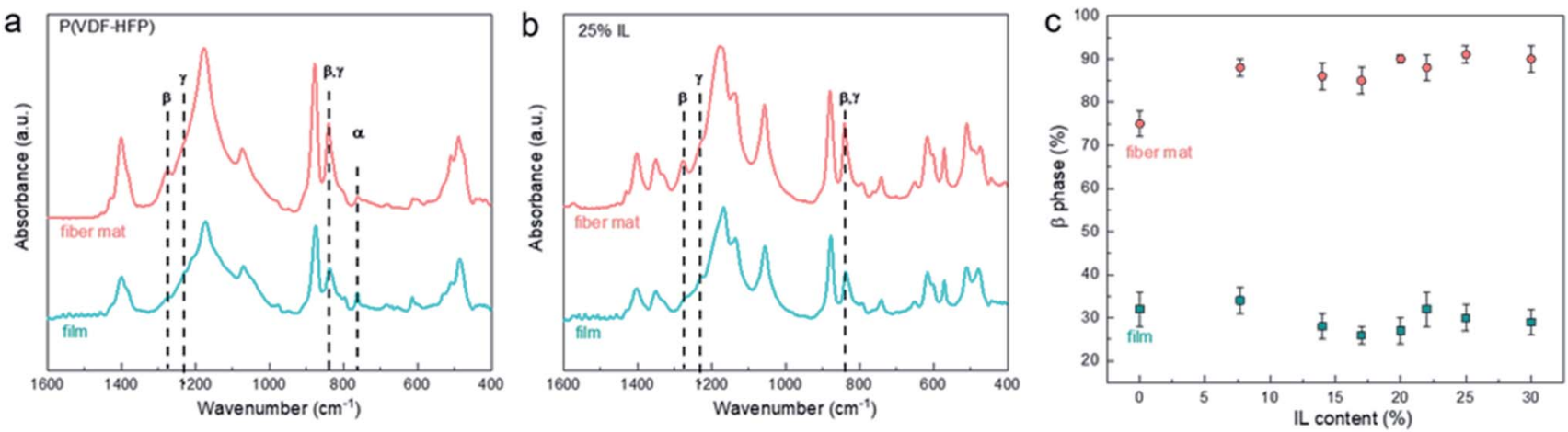

Fig. 2 FT-IR spectra of (a) pure P(VDF-HFP) film and fiber mat. (b) $25 \% \mathrm{IL}$ containing P(VDF-HFP) fiber mat and solution casted film. (c) $\beta$-Phase content of electrospun fiber and films with different IL content. 

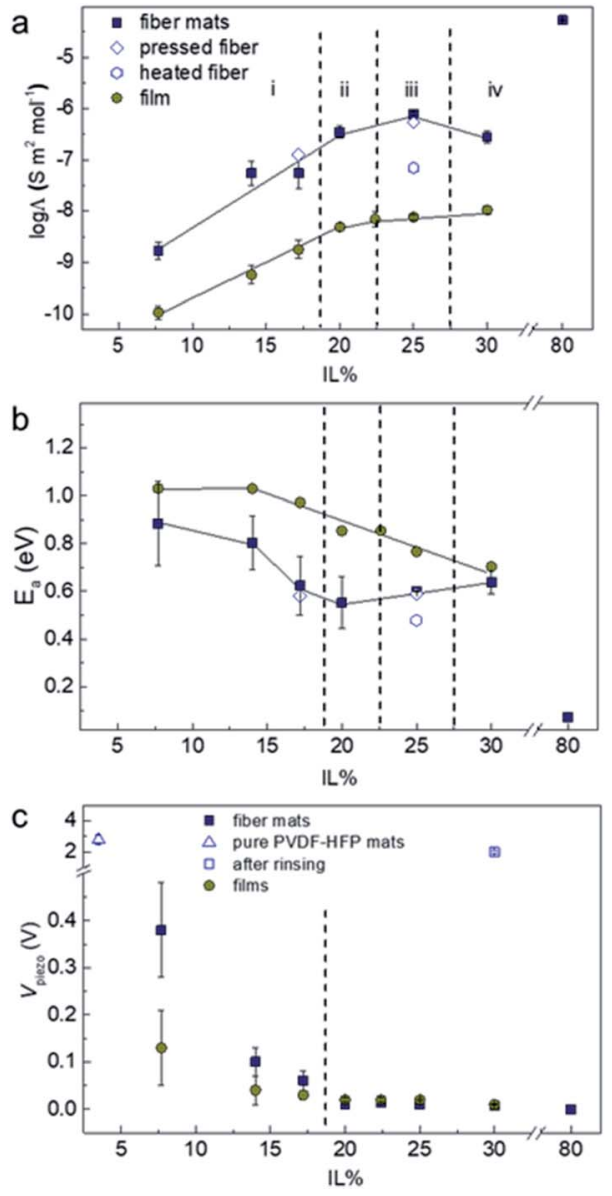

Fig. 3 The ionic transport in the IL-PGs. (a) The room temperature molar conductivity $(\Lambda)$ of the ferroelectric fiber mats compared with the films containing the same percentage of IL. (b) The activation energy of the same samples in (a). (c) Piezoelectric output voltage of all the fiber mats and films.

fiber mats composed of electrospun fibers are one to two orders of magnitude higher than that of the films for all the studied IL content.

In order to better understand the ionic transport mechanism, the activation energy of the composites was obtained by plotting the conductivity at different temperature against the reversed absolute temperature. As shown in Fig. 3b, the activation energy $\left(E_{\mathrm{a}}\right)$ of ferroelectric fiber mats is smaller than that of the films, indicating that less energy is needed for the ions to move in the ferroelectric fiber mats, which agrees with the conductivity results. The activation energy for films is quite similar for small IL content (from $7.7 \%$ to $14 \%$ ) and starts to decrease obviously from $17 \%$. This is in agreement with the increasing trend of their molar conductivity in linear scale (Fig. S5 $\dagger$ ), which increases slowly from $7.7 \%$ to $14 \%$ and faster afterwards. The combination of the activation energy and molar conductivity indicates that the conductivity in films is mostly an effect of mobility of the ions. The main differences of the ferroelectric fiber mats compared to the films are the fibrous morphology and enhanced $\beta$-phase content, where lays the origin for the enhancement in conductivity. To verify the contribution of the morphology and $\beta$-phase content to the conductivity enhancement, the ferroelectric fiber mats were cold pressed or heated respectively, and the conductivity were compared with the original sample. After pressing two of the fiber mats with pressure of five metric tons, the film becomes denser (the SEM image is shown in Fig. S6†) and the porosity decreased from $77 \%$ to around $48 \%$ (same calculation as in ESI, Note $\mathrm{S} 2 \dagger)$. But no significant change in the molar conductivity and $E_{\mathrm{a}}$ (open diamond) confirms that the fiber morphology has trivial effect on the ionic transport. However, the molar conductivity of the fiber mats decreases dramatically after heating at $120^{\circ} \mathrm{C}$. As indicated by the SEM images (Fig. S7 $\dagger$ ) and the FTIR spectroscopy (Fig. S8 $\dagger$ ), both the porosity and polar phase content of the heated fiber mat decreases. It is important to note that although the polar $\beta$-phase of the heated fiber mat reduces from $88 \%$ to $70 \%$, it is still much higher than that of the film $(\sim 30 \%)$. The decreasing $E_{\mathrm{a}}$ value for the heated samples could be related to the fiber merging during the heating while the polar $\beta$-phase content is still high. This proved that the higher ferroelectric phase content in the fiber mat is responsible for the enhanced ionic conductivity in the fibers mats.

As proposed above, we believe that the ferroelectric $\beta$-phase of PVDF in the electrospun fiber mats does affect the ionic conductivity. In order to compare the ferroelectric $\beta$-phase in the fiber mats and drop-casted films, the piezoelectric response of different $\mathrm{P}$ (VDF-HFP) composites were characterized by applying the same force (detailed discussion can be found in ESI, Note S4, Fig. S9-S12 $\dagger$ ). As shown in Fig. S9, $\dagger$ pure P(VDFHFP) fiber mat-based piezoelectric device delivers an output voltage of about $2.5 \mathrm{~V}$ which is comparable with previously reported data in the literatures (see Table S1 $\dagger$ ). The higher piezoelectric response in electrospun $\mathrm{P}(\mathrm{VDF}-\mathrm{HFP})$ fiber matbased device ( $88 \% \beta$-phase) compared to that of the filmbased device (30\% $\beta$-phase) has several origins: firstly, the higher content in $\beta$-phase leading to larger induced dipole orientation. ${ }^{53}$ Secondly, the fiber-mat has a significant porosity ( $\sim 77 \%)$. Hence, we can calculate the effective piezoelectric coefficient $d_{33}$ assuming the volume of the uncompressed fiber mat with a relative dielectric constant $\varepsilon_{\mathrm{r}}=77 \% \varepsilon_{\mathrm{r}}($ air $)+33 \%$ $\varepsilon_{\mathrm{r}}(\mathrm{PVDF})$ with $d_{33}=\frac{Q}{F}=\frac{C V}{F}=\frac{\varepsilon_{0} \varepsilon_{\mathrm{r}} A V}{F d}$, where $Q$ is charge, $F$ is applied force $(24 \mathrm{~N}), V$ is the piezoelectric voltage $(2.5 \mathrm{~V}), C$ is capacitance $=\frac{\varepsilon_{0} \varepsilon_{\mathrm{r}} A}{d}$ with $\varepsilon_{0}-$ vacuum dielectric constant, $\varepsilon_{\mathrm{r}}-$ relative dielectric constant, $A$ - the area of applied force $(1.13 \times$ $\left.10^{-4} \mathrm{~m}^{2}\right), d$ - thickness $(50 \mu \mathrm{m})$. The $d_{33}$ is estimated to be 8.5 pC $\mathrm{N}^{-1}$. Now, we assume the situation where the fiber-mat is fully compressed without air gaps. This is of course the extreme case, likely not achievable, but we consider it to illustrate the limit of the compression. The thickness of the fiber mat will become $33 \%$ of the original thickness $(16.5 \mu \mathrm{m})$ and with relative dielectric constant is $\varepsilon_{\mathrm{r}}=\varepsilon_{\mathrm{r}}(\mathrm{PVDF})$. The piezoelectric coefficient of compressed mat increases significantly to $d_{33}=63 \mathrm{pC}$ $\mathrm{N}^{-1}$. This dimensional effect due to porosity resembles to some of the concept of ferroelectrets in porous polymer foams, ${ }^{54}$ the difference is that ferroelectrets use electrostatic charges, while our porous PVDF fiber mats use permanent dipoles obtained 
from the aligned $\beta$-phase in the nanofibers. Those two reasons support the fact that electrospun PVDF fiber mats usually have higher piezoelectric performance than PVDF films $\left(d_{33} \sim 24-34\right.$ pC $\left.\mathrm{N}^{-1}\right) .{ }^{44}$ Note that a similar device composed of an electrospun fiber mat made of the non-piezoelectric cellulose acetate did not generate any output voltage when the same condition of force was applied (Fig. S10 $\dagger$ ), which excludes the contribution from electrostriction effect, electret effect or triboelectric effect. The correlation of the piezoelectric response to the behavior of ionic conductivity helps us to explore the interaction between the dipoles in PVDF and the ions. As shown in Fig. 3c, the piezoelectric output of the fiber mats in region (i) show typical piezoelectric response when applying pressure. The decreasing output voltage compared to pure $\mathrm{P}(\mathrm{VDF}-\mathrm{HFP})$ fiber indicates the partially screening of the dipoles by adding IL that introduced ionic conduction (Fig. S9, detailed discussion can be found in Note $\mathrm{S} 4 \dagger$ ). While for fiber mats containing IL more than $20 \%$, the piezoelectric response cannot be observed anymore because they are fully screened by the ions. The results shown that the ion-dipole interaction in the ferroelectric fiber mats increases with IL content in region (i) and saturated afterwards. The maximized interaction results in the highest molar conductivity of the fiber mats in the studied IL content range. After removing most of the ionic liquid from the $30 \%$ IL containing fiber mats by rinsing with water, the piezoelectric response recovered back to similar level of pure P(VDF-HFP) fiber mats (the open square in Fig. 3c, and $\mathrm{S} 12 \dagger)$. This proves that the reduction of piezoelectric response with increasing ionic liquid content is due to the screening effect. Moreover, the output voltage of the films in piezoelectric region (region i) is smaller than that of the fiber mats for the same IL content with the same applied force (piezo responses from films are given in Fig. S13 $\dagger$ ). The comparison of the piezoelectric performance between the solution casted films and fiber mats is in agreement with the ferroelectric phase contents characterized by FTIR, which further confirms that the PVDF domains in the fiber mats are indeed polarized by electrospinning. ${ }^{39,55}$

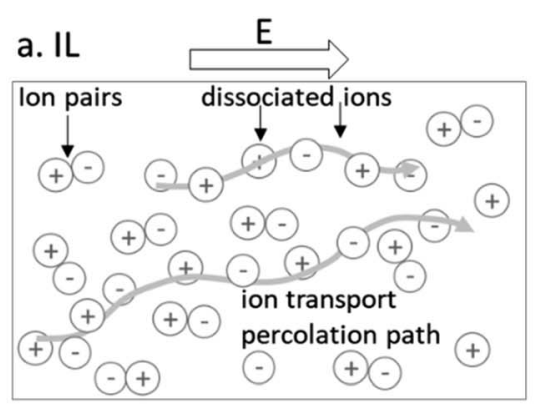

b. P(VDF-HFP)

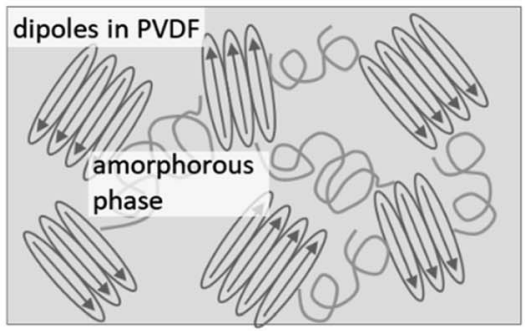

\section{c. $\mathrm{P}(\mathrm{VDF}-\mathrm{HFP})+\mathrm{IL}<22 \%(\mathrm{i} / \mathrm{ii})$}

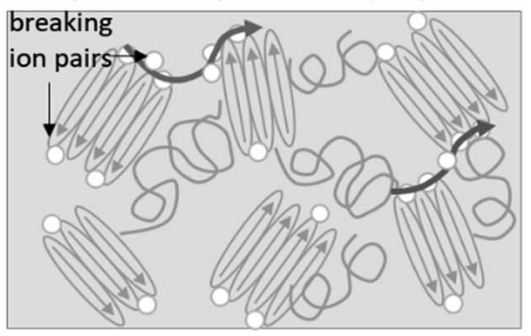

\section{d. $P($ VDF-HFP) $+22 \%<I L<27 \%$ (iii)}

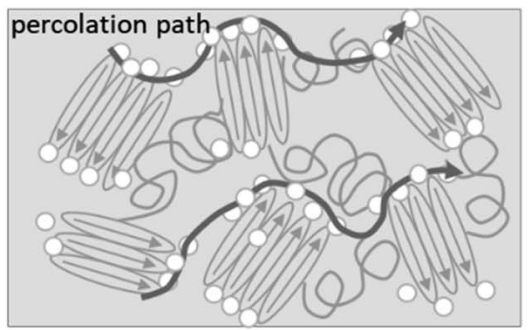

\section{e. $P($ VDF-HFP) $+27 \%<I L$ (iv)}
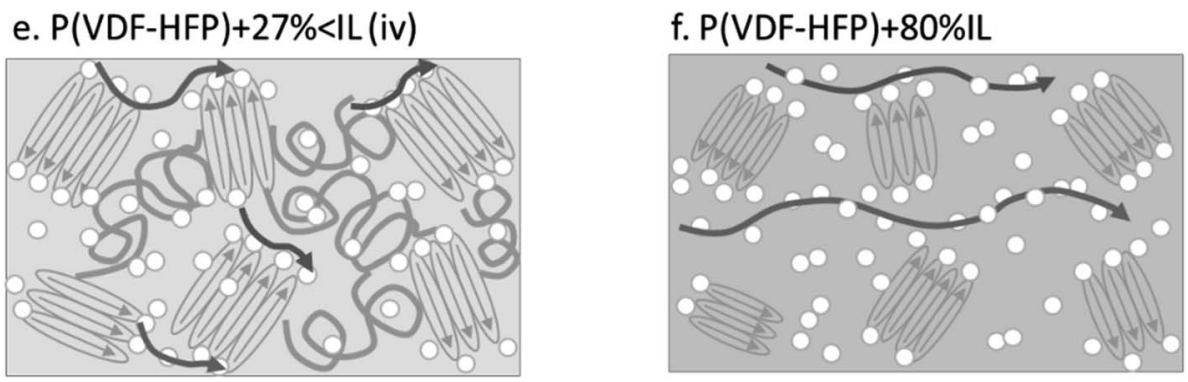

Fig. 4 The illustration of the mechanism of the ion transport in the ferroelectric polymer matrix. (a) In pure ionic liquid, the conduction path is formed through dissociated ions among ion-pairs. $E$ illustrates the direction of electric field in conductivity measurements. (b) P(VDF-HFP) polymer matrix composed of PVDF crystalline phase and HFP amorphous phase. IL will be loaded in the HFP domain. (c) When small amount of IL is loaded in the polymer, the ions interact with PVDF dipoles and ion pairs intend to dissociate. (d) With increasing amount of IL, percolation path is formed through the ions at the interface between crystal and amorphous phase, and conductivity reach maximum. (e) With even more IL, the amorphous phase becomes swollen, and break the connections of the interface percolation path, conductivity decrease. (f) With large amount of IL loading, the amorphous phase is completely soaked with ions, similar percolation path as in pure IL is formed. 


\subsection{Proposed mechanism of ionic conductivity in a ferroelectric medium}

The proposed mechanism for the increased ionic conductivity of the IL in fiber mats compared to the solution casted films is illustrated in Fig. 4. In pure ionic liquid, large percentage of the ions form neutral ion-pairs or neutral aggregations, ${ }^{4}$ and conduction path is connected through dissociated ions (Fig. 4a). The structure of the crystalline and amorphous phase of $\mathrm{P}(\mathrm{VDF}-\mathrm{HFP})$ matrix is illustrated in Fig. $4 \mathrm{~b}$. In the composite of IL and P(VDF-HFP), the ions are soaked in the amorphous HFP domains and can interact with the PVDF dipoles in crystalline phase. When small amount of IL is mixed in the polymer matrix (region i and ii, IL content below 22\%), the molar conductivity of the fiber mats increases due to the increasing amount of mobile ions similar as for the films. Different from the films, $E_{\mathrm{a}}$ of the ferroelectric fiber mats in this concentration region decreases faster. As illustrated in Fig. 4c, this could be due to the electrical interaction between the dipoles and the ions that promotes ion-pair or neutral ionic aggregates dissociation and increases the local mobile ion concentration. ${ }^{56}$ Previous studies have shown that the concentration of IL in PVDF copolymer could be higher at the crystal-amorphous interphase compared to the region of HFP amorphous phase. ${ }^{57}$ In addition, the effect of electric dipoles in a polymer matrix to ionic transport might be comparable to the previously studied charged nanochannels, which are found to promote ionic conductivity in low concentration due to interfacial interactions. ${ }^{58}$

When the IL content in the polymer matrix increases over $\sim 22 \%$ in region (iii), $E_{\mathrm{a}}$ starts to increase for the fiber mats because the additional amount of ions does not interact with the dipoles anymore but behave like in the films. However, the molar conductivity still increases, which indicates that the conductivity in the fiber mats in region (iii) is not only an effect of mobility of the ions (directly related to the activation energy) but also an effect of the concentration of mobile ions. Our hypothesis is that the ion percolation paths are better connected with increasing amount of IL (as shown in Fig. 4d). With further increasing IL content in region (iv), the conductivity starts to decrease and $E_{\mathrm{a}}$ keeps increasing. As shown in Fig. 4e, this is due to the excess number of ions that do not interact with the dipoles swell the amorphous domains and break the connection of the formed percolation path. With even more IL loading (80\%), the conductivity of the fiber mats increases again. At this IL content, the ions completely fill up the pores in the fiber mat and ionic transport path is analogous to that in pure IL (Fig. 4f).

It should be mentioned that PVDF films with high $\beta$-phase content can be achieved by using $\mathrm{P}(\mathrm{VDF}-\mathrm{TrFE})$ instead of $\mathrm{P}(\mathrm{VDF}-$ HFP). The FTIR spectroscopy of the P(VDF-TrFE) films with increasing IL content from $7.7 \%$ to $30 \%$ (same as the P(VDFHFP) films) are presented in Fig. S14. $\dagger$ All the P(VDF-TrFE)/IL films shows strong absorption peaks at $844 \mathrm{~cm}^{-1}$ and $1288 \mathrm{~cm}^{-1}$ that confirms the presence of $\beta$-phase, and no peak featuring the presence of $\alpha$ - or $\gamma$-phases was found. ${ }^{59,60}$ Hence, it is considered that the $\beta$-phase content of these $\mathrm{P}(\mathrm{VDF}-\mathrm{TrFE}) / \mathrm{IL}$ films is $100 \%$ (Fig. 5a) irrespective of the presence of added ionic liquid. ${ }^{\mathbf{4 4 4 9}}$ This is in agreement with previous studies showing that $\mathrm{P}(\mathrm{VDF}-\mathrm{TrFE})$ was specially designed to form spontaneously ferroelectric $\beta$-phase from solution even without poling. ${ }^{59}$ Based on our proposed mechanism, the composite films of $\mathrm{P}(\mathrm{VDF}-\mathrm{TrFE})$ are expected to exhibit higher ionic conductivity compared to $\mathrm{P}(\mathrm{VDF}-\mathrm{HFP})$ in the same IL content range. From Fig. 5b, the conductivity of the $\mathrm{P}(\mathrm{VDF}-\mathrm{TrFE})$ films is indeed much higher than that of films composed of $\mathrm{P}(\mathrm{VDF}-\mathrm{HFP})$ in the whole studied IL content range. As P(VDF-TrFE)/IL films exhibit $100 \% \beta$-phase content, and it also shows higher ionic conductivity compared to P(VDF-HFP)/IL films ( $\beta$-phase content of $\sim 30 \%$ ), we can conclude that the ferroelectric phase content of the polymer matrix is the main contribution of the enhanced ionic conductivity instead of the fiber morphology.

The ionic conductivity of $\mathrm{P}(\mathrm{VDF}-\mathrm{TrFE}) / \mathrm{IL}$ films, $\mathrm{P}(\mathrm{VDF}-\mathrm{HFP}) /$ IL films and $\mathrm{P}(\mathrm{VDF}-\mathrm{HFP}) / \mathrm{IL}$ fiber mats are compared in Fig. S15. $\dagger \mathrm{P}(\mathrm{VDF}-\mathrm{HFP})$ fiber mats have the highest conductivity among the three types of composites, and P(VDF-HFP)/IL films with $30 \%$ of ferroelectric contents have the lowest conductivity. There could be a few reasons to explain the fact that the conductivity of $\mathrm{P}(\mathrm{VDF}-\mathrm{HFP}) / \mathrm{IL}$ fiber mats with $88 \% \beta$-phase content is higher than $\mathrm{P}(\mathrm{VDF}-\mathrm{TrFE}) / \mathrm{IL}$ film with $100 \% \beta$-phase content. Especially note that the electrospun fiber mat is
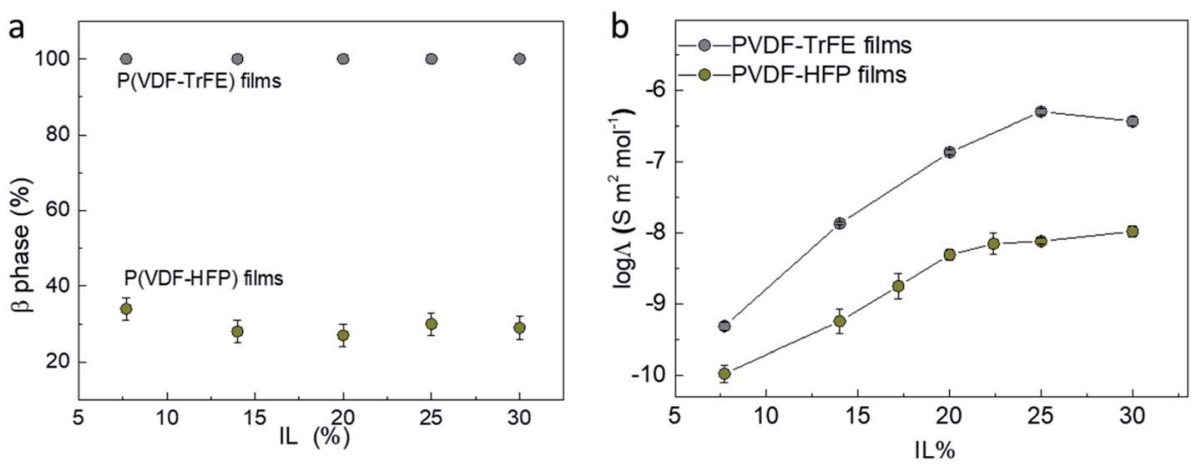

Fig. 5 (a) $\beta$-Phase content of P(VDF-TrFE) and P(VDF-HFP) based solution casted films with different IL content. (b) The molar conductivity of the P(VDF-TrFE) film based ionic liquid gels (solid gray dots) compared with P(VDF-HFP) based films (solid yellow dots) containing the same IL percentage at room temperature. 
a polarized system where $\mathrm{P}(\mathrm{VDF}-\mathrm{TrFE}) / \mathrm{IL}$ film is unpolarized. Previous studies suggest that the structural reorganization with poling process results in improvement of all-trans $\beta$-phase conformation in $\mathrm{P}(\mathrm{VDF}-\mathrm{TrFE})$ crystal structure, ${ }^{61,62}$ though this improvement cannot be evident from vibrational spectra. ${ }^{59,61}$ However, it is difficult to electrically pole $\mathrm{P}(\mathrm{VDF}-\mathrm{TrFE}) / \mathrm{IL}$ film based device with electric field to make a fair comparison with $\mathrm{P}(\mathrm{VDF}-\mathrm{HFP})$ fiber mats due to the presence of ions. The composite sample has low breakdown strength of $20 \mathrm{MV} \mathrm{m}^{-1}$, which is much lower than the voltage $\left(\sim 100 \mathrm{MV} \mathrm{m}^{-1}\right)$ needed to polarize $\mathrm{P}(\mathrm{VDF}-\mathrm{TrFE}) .{ }^{59}$ On the other hand, this highlights the advantage of using electrospinning to spontaneously increase and polarize the ferroelectric $\beta$-phase in PVDF. Furthermore, electrospun $\mathrm{P}(\mathrm{VDF}-\mathrm{HFP})$ fiber mats-based composite also have other advantages compare to $\mathrm{P}(\mathrm{VDF}-\mathrm{TrFE})$ films in terms of material cost.

\subsection{The demonstration of ionic thermometers and temperature mapping}

The activation energy of a conductor is directly related to the conductivity change with temperature, which forms the basic principle for the negative temperature coefficient thermistors (NTC).$^{63}$ Most of the existing NTCs are based on semiconductive metals or ceramics because of their susceptible resistance change with temperature, providing a temperature coefficient of resistance (TCR) of $2.2 \%$ to $5.5 \%{ }^{64}$ The main limit for this type of sensors is that they are bulky and rigid, which are not suitable to be applied in delicate devices. ${ }^{65}$ Here by incorporating the ILPGs, we demonstrated the first organic-based ionic thermistor. From the $E_{\mathrm{a}}$ of the IL-PGs, the TCR can be calculated (ESI, Note
S5 $\dagger) .{ }^{65}$ As shown in Fig. S16, $\dagger$ the AC TCR value of the ferroelectric fiber mats are lower than that of the films, but all of them are between $7.3 \%$ to $13.7 \%$, which are higher than the reported TCR values for NTCs. However, considering the energy consumption proportional to the resistance of the thermistor, the ferroelectric fiber mats containing $25 \%$ IL showing the highest conductivity was chosen to demonstrate temperature mapping function (TCR value of $7.8 \%$ ).

As shown in Fig. 6a, an ionic thermistor array of 4 pixels was fabricated by crossing 2 by 2 fabric electrodes on the two sides of the fiber mat. A temperature gradient was applied across the device by keeping two of the pixels ( 2 and 4 ) on a hot Peltier element and the other two pixels ( 1 and 3 ) on substrate kept at room temperature. The resistance change of the 4 pixels with temperature can be measured from the crossed electrodes. As shown in Fig. 6b, the resistance changes of the 4 pixels followed the temperature profile measured with commercial thermocouple (TC). The smaller and slower resistance changes for pixel 1 and 3 compare to pixel 2 and 4 show that the heat transported to them were less and delayed compare to the directly heated pixels. The enlarge curve in Fig. 6c compares the responding of pixel 2 with the commercial TC. The well overlapped curve shows that the ionic thermistor composed fiber mats responds as fast the TC. This result prove that the ionic thermistor array prepared on the same ferroelectric IL-PG fiber mat responds to temperature change independently and simultaneously. We further demonstrated the temperature mapping using an ionic thermistor composed of 16 pixels. The temperature distribution of the substrate measured by the ionic thermistor and directly with thermocouple are shown in Fig. $6 \mathrm{~d}$ and e. The calculated
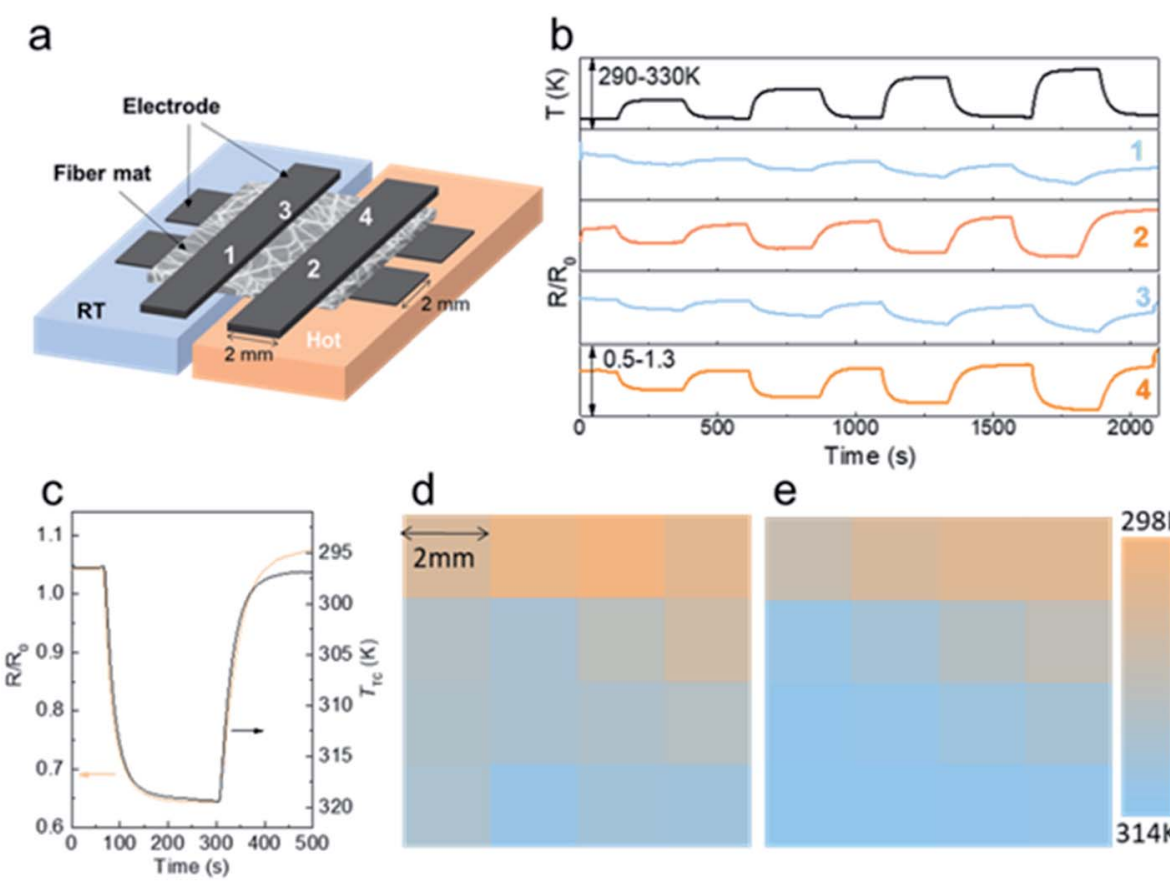

d

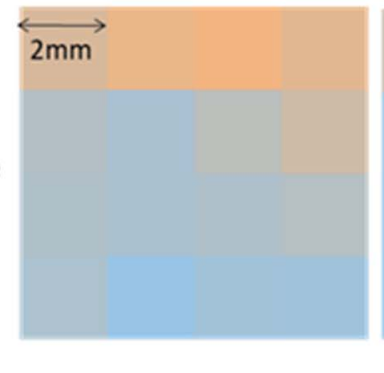

e

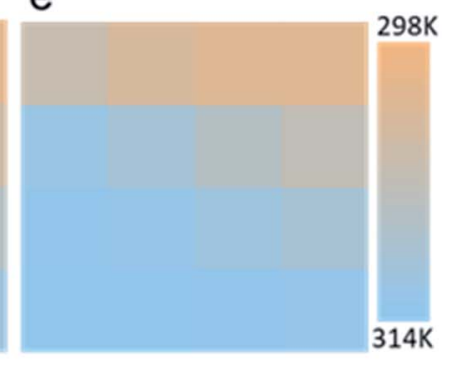

Fig. 6 Demonstrating the ionic thermistor. (a) Schematic diagram for the 4-pixel i-thermistor placed in a temperature gradient. (b) The relative resistance changes of the 4 -pixels with temperature change. (c) The responding comparison between the thermocouple and pixel 2 . (d) The temperature distribution of a surface measured by a $4 \times 4$ ionic thermistor and (e) measured by commercial thermocouple. 
temperature from $\ln \left(R / R_{0}\right)$ matches the experimental temperature distribution, suggesting that the ionic thermistor can successfully map temperature. From the results of ionic thermistor performance demonstration recorded by Keithley analyzer, the TCR is calculated to be $3.10 \pm 0.05 \%$ at room temperature.

\section{Conclusion}

We studied solid-state electrolytes made of an ionic liquid blended in a ferroelectric copolymer of PVDF. By using electrospinning technology, we succeeded to increase the ferroelectric phase content in PVDF with the presence of ionic liquids. The piezoelectric responses of the electrospun fiber mats decreases with the amount of ionic liquids, but are superior than casted films of the same composite. We found that the molar ionic conductivity in the ferroelectric mats was 100 times higher than that in the films. The observation combined with FTIR analysis, activation energy for the ionic transport and piezoelectric voltage response, offer us a consistent model to explain this key observation and confirm that the enhancement in conductivity is mainly due to the ion-dipole interaction in the ferroelectric mats. Hence, we have succeeded to enhance the ionic conductivity of ionic liquid by the presence of ferroelectric $\beta$-phase content of PVDF based polymer.

Taking advantage of the enhanced ionic conductivity and high temperature coefficient, we fabricated a thin and flexible ionic thermistor with a TCR of $3 \%$ in DC operation and $7.8 \%$ in AC characterization. The simple structure of the thin film ionic thermistor enables facile fabrication of multi-pixel sensors that can monitor temperature distribution synchronously. Polymeric ionic conductors have potential to achieve good charge carrier transport, sufficient mechanical strength, and flexibility, which are important characters in high-tech research fields. This method of enhancing ionic conductivity provides a new perspective in engineering electrolytes for future applications.

\section{Experimental section}

\subsection{Materials}

Poly(vinylidene fluoride-hexafluoropropylene) P(VDF-HFP), $\left(M_{\mathrm{W}}\right.$ $=400000), \quad$ poly(vinylidene fluoride-cotrifluoroethylene) (P(VDF-TrFE)) (70:30 mol\%), N,N-dimethylformamide (DMF) and 1-ethyl-3-methylimidazolium bis(trifluoromethylsulfonyl) imide ([EMIM][TFSI]) (Sigma-Aldrich). Tin copper silver plated polyamide ripstop fabric (Kitronik Ltd).

\subsection{P(VDF-HFP)/EMIM TFSI electrospun membranes preparation}

For preparing the electrospun nanofiber membranes at first 12 (w/v\%) of $\mathrm{P}(\mathrm{VDF}-\mathrm{HFP})$ pellets was dissolved in a mixed solvent of DMF and acetone $(3: 2 \mathrm{v} / \mathrm{v})$. The mixture was then stirred for $4 \mathrm{~h}$ at room temperature. When a homogeneous solution was formed EMIM TFSI was mixed to the solution with different weight ratio of the P(VDF-HFP) and EMIM TFSI and stirred at room temperature for $1 \mathrm{~h}$. The different solutions were then used to make electrospun fibers. The solutions were poured into a syringe. A pump was delivering the solutions to a needle with diameter of $\sim 0.8 \mathrm{~mm}$. During the electrospinning of different solutions, the flow rate was 0.2 to $0.4 \mu \mathrm{min}^{-1}$, the voltage was 17 to $19 \mathrm{kV}$ and the needle to collector distance was fixed at $10 \mathrm{~cm}$. Electrospun fibers for only P(VDF-HFP) was also prepared. All the fibers were collected at a grounded aluminum collector. The different P(VDF-HFP)/IL composite fibers are named according to the weight percentage of IL such as $7.7 \%$, $14 \%, 17 \%, 20 \%, 22 \%, 25 \%$ and $30 \%$. A fiber mat with high IL content $(80 \% \mathrm{w} / \mathrm{w})$ was also prepared by adding more IL after preparation of $30 \%$ IL containing fiber mat.

$\mathrm{P}(\mathrm{VDF}-\mathrm{HFP}) / \mathrm{IL}$ composite films were also prepared by solution casting using the same solutions. The different solutions were casted on clean glass slides and dried at $90{ }^{\circ} \mathrm{C}$ for $6 \mathrm{~h}$. After peeling off the free-standing composite films were obtained. They were also named as the same as the fibers.

$\mathrm{P}(\mathrm{VDF}-\mathrm{TrFE}) / \mathrm{IL}$ composite films were also prepared as the same process of $\mathrm{P}(\mathrm{VDF}-\mathrm{HFP}) / \mathrm{IL}$ composite films preparation followed by solution casting on substrates and drying.

\subsection{Device fabrication}

The different electrospun fibers were peeled off from the aluminum foil and sandwiched between two fabric electrodes and finally laminated with $75 \mu \mathrm{m}$ thick lamination paper. The final devices were used for conductivity measurement and ionic thermistor demonstration. The area of the devices are around 1 $\mathrm{cm}^{2}$ and the thickness is around $50 \mu \mathrm{m}$ (the dimension of each device was measured and used in the calculation). Device fabrication process for the films is same as the fibers. The device for temperature mapping was prepared by using stripe shaped fabric electrodes with the width of $2 \mathrm{~mm}$ and the distance between two electrodes is $1 \mathrm{~mm}$.

\subsection{Characterization}

A field-emission scanning electron microscope (SEM) (Sigma 500 Gemini, Zeiss) operated with an acceleration voltage of $3 \mathrm{kV}$ was used to investigate the morphology of the electrospun fibers. The crystallographic phase for the electrospun fiber membranes and solution casted films were identified with Fourier Transform Infrared Spectroscopy (FT-IR) (Bruker, Equinox 55). The impedance measurement was carried out with impedance spectrometer (Alpha high-resolution dielectric analyzer, Novocontrol Technologies $\mathrm{GmbH}$, Hundsangen, Germany) with an AC voltage of $10 \mathrm{mV}$ in the frequency range of 0.1 $\mathrm{HZ}$ to $1 \mathrm{MHz}$. A Keithley analyzer (2400) was used to measure the electrical properties of the ionic thermistor. The piezoelectric response from the devices were tested and recorded with National Instrument (NI) systems. The logging system is NI CDAQ 9174 chassis with NI 9263 voltage output and NI 9239 DAC modules interfaced with a computer using LabVIEW software.

\subsection{Piezoelectric test}

The piezoelectric test was done for all the electrospun fiber and solution cased film based devices under same applied force of $24 \mathrm{~N}$ 
and impact frequency of $3 \mathrm{~Hz}$ by controlling the amplitude and frequency of the shaker. The area of impact is $1.13 \times 10^{-4} \mathrm{~m}^{2}$.

\section{Conflicts of interest}

There are no conflicts to declare.

\section{Acknowledgements}

The authors thank the Knut and Alice Wallenberg Foundation (proof of concept "Hi-VAE"), Swedish Research Council VR 2016-05990 and 2018-04037, Wallenberg Wood Science Center and the Advanced Functional Materials Center at Linköping University (2009-00971).

\section{References}

1 L. Herlogsson, X. Crispin, N. D. Robinson, M. Sandberg, O.-J. Hagel, G. Gustafsson and M. Berggren, Adv. Mater., 2007, 19, 97-101.

2 D. Zhao, S. Fabiano, M. Berggren and X. Crispin, Nat. Commun., 2017, 8, 14214.

3 D. Zhao, H. Wang, Z. U. Khan, J. C. Chen, R. Gabrielsson, M. P. Jonsson, M. Berggren and X. Crispin, Energy Environ. Sci., 2016, 9, 1450-1457.

4 D. Zhao, A. Martinelli, A. Willfahrt, T. Fischer, D. Bernin, Z. U. Khan, M. Shahi, J. Brill, M. P. Jonsson, S. Fabiano and X. Crispin, Nat. Commun., 2019, 10, 1093.

5 H. Cheng, X. He, Z. Fan and J. Ouyang, Adv. Energy Mater., 2019, 9, 1901085.

6 J. Wang, C. Yan, G. Cai, M. Cui, A. L.-S. Eh and P. S. Lee, Adv. Mater., 2016, 28, 4490-4496.

7 M. He, K. Zhang, G. Chen, J. Tian and B. Su, ACS Appl. Mater. Interfaces, 2017, 9, 16466-16473.

8 B. Alberts, D. Bray, J. Watson, J. Lewis, K. Roberts and M. Raff, Molecular Biology of the Cell, Garland Science, New York, 4th edn, 2002.

9 T. A. Sjöström, M. Berggren, E. O. Gabrielsson, P. Janson, D. J. Poxson, M. Seitanidou and D. T. Simon, Adv. Mater. Technol., 2018, 3, 1700360.

10 T. Li, L. Li, H. Sun, Y. Xu, X. Wang, H. Luo, Z. Liu and T. Zhang, Adv. Sci., 2017, 4, 1600404.

11 G. Liang, Z. Ruan, Z. Liu, H. Li, Z. Wang, Z. Tang, F. Mo, Q. Yang, L. Ma, D. Wang and C. Zhi, Adv. Electron. Mater., 2019, 5, 1900553.

12 M. A. Darabi, A. Khosrozadeh, R. Mbeleck, Y. Liu, Q. Chang, J. Jiang, J. Cai, Q. Wang, G. Luo and M. Xing, Adv. Mater., 2017, 29, 1700533.

13 Q. Pan, D. Barbash, D. M. Smith, H. Qi, S. E. Gleeson and C. Y. Li, Adv. Energy Mater., 2017, 7, 1701231.

14 A. M. Oickle and H. A. Andreas, J. Phys. Chem. C, 2011, 115, 4283-4288.

15 E. Said, O. Larsson, M. Berggren and X. Crispin, Adv. Funct. Mater., 2008, 18, 3529-3536.

16 N. Plumeré, O. Rüdiger, A. A. Oughli, R. Williams, J. Vivekananthan, S. Pöller, W. Schuhmann and W. Lubitz, Nat. Chem., 2014, 6, 822-827.
17 A. Yella, H.-W. Lee, H. N. Tsao, C. Yi, A. K. Chandiran, M. K. Nazeeruddin, E. W.-G. Diau, C.-Y. Yeh, S. M. Zakeeruddin and M. Grätzel, Science, 2011, 334, 629634.

18 Y. Liu, Y. Zhu and Y. Cui, Nat. Energy, 2019, 4, 540-550.

19 C. Zhong, Y. Deng, W. Hu, J. Qiao, L. Zhang and J. Zhang, Chem. Soc. Rev., 2015, 44, 7484-7539.

20 A. Arya and A. L. Sharma, Ionics, 2017, 23, 497-540.

21 J.-H. Shin, W. A. Henderson and S. Passerini, Electrochem. Commun., 2003, 5, 1016-1020.

22 I. Osada, H. d. Vries, B. Scrosati and S. Passerini, Angew. Chem., Int. Ed., 2016, 55, 500-513.

23 B. Yang, C. Li, J. Zhou, J. Liu and Q. Zhang, Electrochim. Acta, 2014, 148, 39-45.

24 C. Arbizzani, G. Gabrielli and M. Mastragostino, J. Power Sources, 2011, 196, 4801-4805.

25 Y.-S. Ye, J. Rick and B.-J. Hwang, J. Mater. Chem. A, 2013, 1, 2719-2743.

26 H. Tsutsumi, A. Matsuo, K. Takase, S. Doi, A. Hisanaga, K. Onimura and T. Oishi, J. Power Sources, 2000, 90, 33-38.

27 D. Lin, P. Y. Yuen, Y. Liu, W. Liu, N. Liu, R. H. Dauskardt and Y. Cui, Adv. Mater., 2018, 30, 1802661.

28 S. Abbrent, J. Plestil, D. Hlavata, J. Lindgren, J. Tegenfeldt and Å. Wendsjö, Polymer, 2001, 42, 1407-1416.

29 T. E. Sutto, J. Electrochem. Soc., 2007, 154, P101-P107.

30 T. E. Sutto, J. Electrochem. Soc., 2007, 154, P130-P135.

31 T. Fukushima, K. Asaka, A. Kosaka and T. Aida, Angew. Chem., Int. Ed., 2005, 44, 2410-2413.

32 S. Ferrari, E. Quartarone, P. Mustarelli, A. Magistris, M. Fagnoni, S. Protti, C. Gerbaldi and A. Spinella, J. Power Sources, 2010, 195, 559-566.

33 J. C. Dias, D. C. Correia, A. C. Lopes, S. Ribeiro, C. Ribeiro, V. Sencadas, G. Botelho, J. M. S. S. Esperanca, J. M. Laza, J. L. Vilas, L. M. León and S. L. Méndez, J. Mater. Sci., 2016, 51, 4442-4450.

34 D. Rollik, S. Bauer and R. Gerhard-Multhaupt, J. Appl. Phys., 1999, 85, 3282-3288.

35 D. Mandal, S. Yoon and K. J. Kim, Macromol. Rapid Commun., 2011, 32, 831-837.

36 A. Laforgue, L. Robitaille, A. Mokrini and A. Ajji, Macromol. Mater. Eng., 2007, 292, 1229-1236.

37 X. Li, X. Hao, D. Xu, G. Zhang, S. Zhong, H. Na and D. Wang, J. Membr. Sci., 2006, 281, 1-6.

38 B. Dong, L. Gwee, D. S. Cruz, K. I. Winey and Y. A. Elabd, Nano Lett., 2010, 10, 3785-3790.

39 J. Fang, X. Wang and T. Lin, J. Mater. Chem., 2011, 21, 1108811091.

40 D. Mandal, K. Henkel and D. Schmeißer, Phys. Chem. Chem. Phys., 2014, 16, 10403-10407.

41 D. H. Reneker, A. L. Yarin, H. Fong and S. Koombhongse, J. Appl. Phys., 2000, 87, 4531-4547.

42 J. M. Seo, G. K. Arumugam, S. Khan and P. A. Heiden, Macromol. Mater. Eng., 2009, 294, 35-44.

43 A. Sultana, P. Sadhukhan, M. M. Alam, S. Das, T. R. Middya and D. Mandal, ACS Appl. Mater. Interfaces, 2018, 10, 41214130. 
44 P. Martins, A. C. Lopes and S. Lanceros-Mendez, Prog. Polym. Sci., 2014, 39, 683-706.

45 K. Castkova, J. Kastyl, D. Sobola, J. Petrus, E. Stastna, D. Riha and P. Tofel, Nanomaterials, 2020, 10, 1221.

46 Y. Bormashenko, R. Pogreb, O. Stanevsky and E. Bormashenko, Polym. Test., 2004, 23, 791-796.

47 A. Sultana, M. M. Alam, P. Sadhukhan, U. K. Ghorai, S. Das, T. R. Middya and D. Mandal, Nano Energy, 2018, 49, 380-392.

48 M. M. Alam, A. Sultana, D. Sarkar and D. Mandal, Nanotechnology, 2017, 28, 365401.

49 J. C. Dias, D. M. Correia, C. M. Costa, C. Ribeiro, A. Maceiras, J. L. Vilas, G. Botelho, V. Z. Bermudez and S. L. Mendez, Electrochim. Acta, 2019, 296, 598-607.

50 X. Pan, Z. Wang, Z. Cao, S. Zhang, Y. He, Y. Zhang, K. Chen, Y. Hu and H. Gu, Smart Mater. Struct., 2016, 25, 105010.

51 M. A. Ratner and D. F. Shriver, Chem. Rev., 1988, 88, 109-124.

52 M. Patel, K. G. Chandrappa and A. J. Bhattacharyya, Solid State Ionics, 2010, 181, 844-848.

53 M. G. Broadhurst, G. T. Davis and J. E. McKinney, J. Appl. Phys., 1978, 49, 4992-4997.

54 S. Bauer, R. G. Multhaupt and G. M. Sessler, Phys. Today, 2004, 57, 37-43.
55 X. Liu, S. Xu, X. Kuang, D. Tan and X. Wang, RSC Adv., 2016, 6, 109061-109066.

56 H. Y. Sun, H.-J. Sohn, O. Yamamoto, Y. Takeda and N. Imanishi, J. Electrochem. Soc., 1999, 146, 1672-1676.

57 C. Xing, M. Zhao, L. Zhao, J. You, X. Cao and Y. Li, Polym. Chem., 2013, 4, 5726-5734.

58 C. Duan and A. Majumdar, Nat. Nanotechnol., 2010, 5, 848852.

59 X. Qiu, G. C. Schmidt, P. M. Panicker, R. A. Q. Soler, A. J. Benjamin and A. C. Hübler, Adv. Eng. Mater., 2019, 21, 1900537.

60 A. Mayeen, M. S. Kala, M. S. Jayalakshmy, S. Thomas, D. Rouxel, J. Philip, R. N. Bhowmik and N. Kalarikkal, Dalton Trans., 2018, 47, 2039-2051.

61 K. J. Kim and G. B. Kim, Polymer, 1997, 38, 4881-4889.

62 F. Fang, W. Yang, C. Jia and X. Luo, Appl. Phys. Lett., 2008, 92, 222906.

63 M. Lee and M. Yoo, Sens. Actuators, A, 2002, 96, 97-104.

64 A. Feteira, J. Am. Ceram. Soc., 2009, 92, 967-983.

65 C. Yan, J. Wang and P. S. Lee, ACS Nano, 2015, 9, 2130-2137. 